\title{
Chemical Imaging Analysis of Active Pharmaceutical Ingredient in Dissolving Microneedle Arrays By Raman Spectroscopy
}

Daisuke Ando ( $\square$ ando-daisuke@nihs.go.jp )

National Institute of Health Sciences https://orcid.org/0000-0001-6683-4874

Tamaki Miyazaki

National Institute of Health Sciences

Eiichi Yamamoto

National Institute of Health Sciences

Tatsuo Koide

National Institute of Health Sciences

Ken-ichi Izutsu

National Institute of Health Sciences

\section{Research Article}

Keywords: Dissolving microneedles, Raman spectroscopy, drug distribution, drug delivery system, quality 7 evaluation method

Posted Date: April 13th, 2021

DOl: https://doi.org/10.21203/rs.3.rs-359979/v1

License: (9) (1) This work is licensed under a Creative Commons Attribution 4.0 International License. Read Full License

Version of Record: A version of this preprint was published at Drug Delivery and Translational Research on August 24th, 2021. See the published version at https://doi.org/10.1007/s13346-021-01052-y. 
[Title]

2 Chemical imaging analysis of active pharmaceutical ingredient in dissolving microneedle

3 arrays by Raman spectroscopy

4

5 [Authors]

6 Daisuke Ando*, Tamaki Miyazaki, Eiichi Yamamoto, Tatsuo Koide, Ken-ichi Izutsu

8 [Affiliation]

9 Division of Drugs, National Institute of Health Sciences, 3-25-26 Tonomachi, Kawasaki-ku, Kawasaki,

10 Kanagawa, 210-9501, Japan

\section{2 [Correspondent Footnote]}

13 Daisuke Ando, Ph.D.

14 Division of Drugs, National Institute of Health Sciences, 3-25-26 Tonomachi, Kawasaki-ku, Kawasaki,

15 Kanagawa, 210-9501, Japan

16 Tel.: +81-44-270-6509

$17 \quad$ Fax.: +81-44-270-6511

18 E-mail address: ando-daisuke@nihs.go.jp 


\section{Abstract}

The purpose of this study was to develop a quality evaluation method for dissolving microneedle arrays

3

(DMNAs) and determine the spatial distribution pattern of drugs in DMNAs. Raman spectroscopy mapping was used to visualize the drug distribution in DMNAs and drug-loaded polymer films as a model.

Powder X-ray diffraction (PXRD) and high-pressure liquid chromatography were also performed to characterize DMNAs. Drug-loaded polymer films and DMNAs were prepared by drying the aqueous solutions spread on the plates or casting. PXRD analysis suggested the crystallization of diclofenac sodium (DCF) in several forms depending on its amount in the sodium hyaluronate (HA)-based films.

The Raman spectra of HA and DCF showed characteristic and non-overlapping peaks at 1376 and 1579 $\mathrm{cm}^{-1}$ Raman shifts, respectively. The intensity of the characteristic peak of DCF in the DCF-loaded films increased linearly with the increasing drug content in the range of 4.8 to $16.7 \%$ (DCF, w/w). Raman imaging analysis revealed a homogenous dispersion of small DCF crystals in these films. Raman imaging indicates the distribution of DCF on the surface of the DMNA needle. This work highlights the benefit of using Raman spectroscopy mapping to reveal the spatial distribution of drugs in DMNAs. 


\section{Optical image}

Sodium hyaluronate

\section{Diclofenac sodium}

\section{2 components}

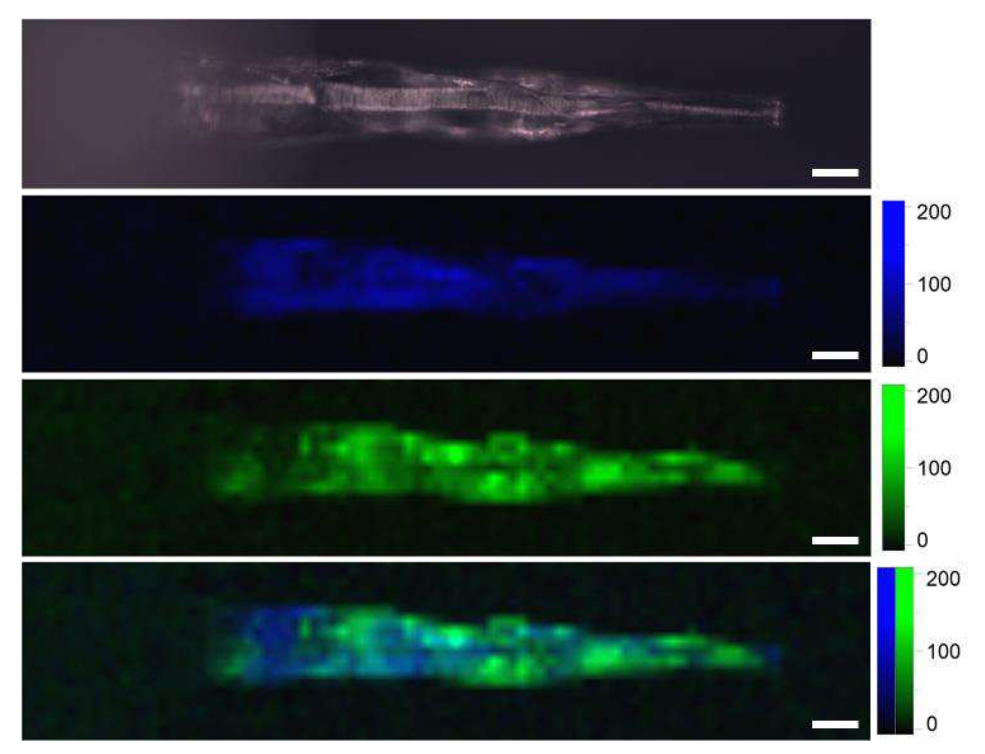

2

$6 \quad$ [Keywords]

7 Dissolving microneedles, Raman spectroscopy, drug distribution, drug delivery system, quality

8 evaluation method

9

10

11 
1

2

\section{Introduction}

The transdermal drug delivery system (TDDS) is an attractive alternative to conventional drug delivery methods of oral administration or injection. TDDS offers a variety of advantages, including feasible controlled delivery or sustained release of drugs, avoiding first-pass hepatic metabolism, and a patient-friendly approach [1]. However, molecular transdermal transport is limited by the skin barrier function, which forms epidermal tight junctions. Microneedle arrays (MNAs) [2] are an effective approach to deliver chemical therapeutics, biologicals, and vaccine antigens from the skin, overcoming these limitations. MNAs are minimally invasive drug delivery systems that painlessly pierce the stratum corneum (SC) to enhance molecular transdermal transport. MNA is composed of multiple micron-scale needles less than $1 \mathrm{~mm}$ in length, which is long enough to physically penetrate the SC, but short and narrow enough to avoid stimulating pain receptors. MNAs are categorized into four main types as follows: 1) Solid MNAs [3] for skin pretreatment to enhance permeability; 2) hollow MNAs [4] for drug solution injection; 3) coated MNAs [5] with drug coating that dissolves in the skin; 4) dissolving MNAs [6] (DMNA) that encapsulate the drug and dissolve it in the skin. Each type of MNA has specific advantages and disadvantages. The first three types are mainly fabricated with non-biodegradable materials, such as silicon or metal, owing to their stiffness and molding properties [7]. The preparation of DMNAs by using certain dissolving biodegradable materials, such as hyaluronic acid [6], chondroitin sulfate [8], and carboxymethylcellulose [9], as base polymers are considered as promising methods over other types of MNAs, owing to the lower risk of broken needle tip being left in the skin [10], lower 
1 production costs, better stability of loaded active pharmaceutical ingredients (APIs) [11, 12], one-step

2 application, and ability to control the drug release profile [13].

For DMNAs, it is important to determine the spatial distribution of drugs on individual needles [14,

4

15]. While most DMNAs are composed of a needle and a baseplate, only the drug encapsulated at a certain position in the needles would be efficiently delivered via dissolution after application. Methods to prepare DMNAs with appropriate drug distribution and their access have been receiving increasing attention [16]. The preparation methods include the application of certain micromolding technologies. For example, the fabrication of needles using polymer-drug blends, followed by the addition of a baseplate by casting a drug-free formulation decreases API waste $[17,18]$. These preparation methods, however, have some risks as the clinically available dose is unclear due to a possible shift of APIs into the baseplate via diffusion [19]. Despite the importance of accurate drug positioning in DMNAs, appropriate methods to detect the spatial distribution of the drug are not yet available. Preparation of model DMNAs by using a color tracer (Rhodamine B) suggested a large effect of the polymer concentration in the casting solution on the distribution of the tracer [20]. High-pressure liquid chromatography (HPLC) of the drug using the collected needle parts provided only rough information on the content. Therefore, an appropriate method to analyze drug distribution on the needle is warranted. Raman spectroscopy, which provides the molecular fingerprint by measuring the vibrational or rotational energies of chemical bonds, is a potent analytical technology for chemical identification and physical characterization of materials. It has been used as a rapid non-destructive method to analyze 
1 various pharmaceutical formulations and as a process analytical technology tool [21]. In addition,

2 obtaining the Raman spectra at each position of the formulation provides information on the spatial

3 distribution of components, as well as their physical states, which are valuable for troubleshooting

4 manufacturing processes [22], monitoring blend homogeneity [23], revealing the internal structure [24]

5 and understanding the physical attributes [25] of the products.

6 The overall aim of this study was to evaluate the feasibility, methodology, and possible future

7 applications of Raman spectroscopy for the quality evaluation of DMNAs. Herein, we report the

8 chemical imaging of a drug in sodium hyaluronate (HA)-based films and drug-loaded DMNAs using

9 Raman microscopy. We selected diclofenac sodium (DCF) as a model compound. As a widely used non-

10 steroidal anti-inflammatory drug (NSAID), DCF can help compare the advantages and limitations of

11 transdermal systems, including safety and ability to penetrate through the skin and achieve therapeutic

12 concentrations in deep subcutaneous tissues [26].

\section{Materials and methods}

\subsection{Materials}

Sodium hyaluronate (HA) (FCH-SU, lot: HGV016900, average molecular weight (MW): 50,000110,000 Da) was purchased from Kikkoman Biochemifa Company (Tokyo, Japan). Diclofenac sodium

(DCF) was purchased from FUJIFILM Wako Pure Chemical Corporation (Osaka, Japan).

Polydimethylsiloxane (PDMS, Dowsil Silpot 184) was purchased from Dow Corning (Midland, MI, 
1 USA). Aluminum microneedle mold masters, fabricated via micro-milling, were purchased from Tokai

2 Azumi Techno (Mie, Japan). All other chemicals were purchased commercially as reagent-grade

3 products. Water was deionized and purified using a Milli-Q ${ }^{\circledR}$ TOC purification system (Millipore,

4 Bedford, MA, USA).

5

6 2.2. Preparation of HA films

7 We used HA as a base polymer because it is a component of the skin tissue with a hydrophilic nature;

8 thus, it may be biocompatible with the skin and safe for DMNA insertion [6, 11, 27]. DCF was dissolved

9 in distilled water, and then $10 \%(\mathrm{w} / \mathrm{w})$ HA was added and uniformly mixed. Each solution was poured

10 into a silicone rubber mold consisting of a circular area with a diameter of $14 \mathrm{~mm}$. To allow the solution

11 to spread evenly, the mold was placed on a flat surface and dried in a desiccator at room temperature for

$1224 \mathrm{~h}$.

\subsection{DMNA fabrication}

15 DMNA was fabricated using micromolding technology with HA as the base polymer. First, the microneedle mold masters were prepared with the following geometry: 316 conical microneedles, 140 $\mu \mathrm{m}$ base diameter, $40 \mu \mathrm{m}$ tip radius, and $800 \mu \mathrm{m}$ height. Next, inverse replication of the master structures was reproduced using PDMS. The DCF-containing HA solution was prepared in the same manner as the HA films. The aqueous solution was poured into PDMS micro molds. The molds were then 
1

centrifuged at 3,500 rpm for $15 \mathrm{~min}$ at room temperature to allow the solution to fill the holes. After that, the molds were kept for $48 \mathrm{~h}$ at $35^{\circ} \mathrm{C}$ to dry the needles. The DMNA was obtained by separating them from the molds and cutting them into circles of $14 \mathrm{~mm}$ in diameter using a punch. The morphology of DMNA was investigated using a Keyence VHX-5000 digital microscope (Keyence, Osaka, Japan).

\subsection{Quantification of the DCF content}

The DCF content was determined using HPLC. In the case of DMNA, we carefully cut the microneedles from the baseplate with a razor and collected them in $1.5 \mathrm{~mL}$ tubes. Microneedles for each array were then soaked in $1.0 \mathrm{~mL}$ distilled water followed by vortex mixing to completely dissolve them. After appropriate dilution, the sample solution was analyzed using HPLC by referring to the Japanese Pharmacopeia 18 diclofenac sodium suppositories. Briefly, an LC-20A HPLC system (Shimadzu, Kyoto, Japan) with an Inertsil ODS-3 column $(4.0 \times 150 \mathrm{~mm}, 5-\mu \mathrm{m}$ particle size $)$. The mobile phase comprised $0.1 \mathrm{M}$ sodium acetate trihydrate and methanol $(40: 60, \mathrm{v} / \mathrm{v})$. The flow rate and column temperature were $1.1 \mathrm{~mL} / \mathrm{min}$ and $25^{\circ} \mathrm{C}$, respectively, and the injection volume was $20 \mu \mathrm{L}$. HPLC was performed at a detection wavelength of $254 \mathrm{~nm}$. The concentration of DCF in the sample solution was determined using a standard curve based on the known concentration of DCF, which was converted to the absolute mass of DCF loaded into the DMNA with a dilution factor. 

diffractometer (MiniFlex 600, Rigaku, Tokyo, Japan). The sample was spread on a non-reflective plate. The diffraction angle, scan rate, voltage, and current of the generator were set to 2.0 to $40.0^{\circ}, 4.0^{\circ} / \mathrm{min}$, $40 \mathrm{kV}$ and $15 \mathrm{~mA}$, respectively.

\subsection{Raman fiber probe analysis}

A Raman fiber probe was used to detect the Raman spectra of the samples. An iHR320 spectrometer system (Horiba, Kyoto, Japan) with a CCD detector (Syncerity Scientific Cameras, Horiba) was used.

A $100 \mathrm{~mW}, 785 \mathrm{~nm}$ excitation laser was connected to the incidence fiber, while the Raman signal was collected through a 0.5-inch diameter fiber ball probe (Marqmetrix, Seattle, WA, USA). Raman spectra were collected four times at an acquisition time $90 \mathrm{~s}$ by directly touching the samples with the probe head. The relative Raman intensity ratio of the DCF characteristic peak at $1579 \mathrm{~cm}^{-1}$ to the HA characteristic peak at $1376 \mathrm{~cm}^{-1}$ was determined as an internal control using the equation below:

The relative Raman intensity ratio of DCF $=\frac{\text { DCF characteristic peak intensity at } 1579 \mathrm{~cm}^{-1}}{\text { HA characteristic peak intensity at } 1376 \mathrm{~cm}^{-1}}$

\subsection{Confocal Raman microscopy}


1 case of HA films, a $50 \mu \mathrm{m}$ step size was used with an exposure time of $4 \mathrm{~s}$, scanning through a 2,000

$2 \mu \mathrm{m}$ square area (total 1681 points). In the case of DMNA, a $10 \mu \mathrm{m}$ step size was used with an exposure

3 time of $4 \mathrm{~s}$, scanning through a $180 \times 900 \mu \mathrm{m}$ square area (total 1729 points). Raman signatures of the

4 samples were acquired in the fingerprint region $\left(800-2100 \mathrm{~cm}^{-1}\right)$. LabSpec 6.0 software was used for

5 spectral collection, spectral preprocessing, and data set processing. The obtained spectra were

6 preprocessed to remove the influence of fluorescence, and then the spectra were used to generate

7 chemical images using the classical least-squares (CLS) algorithm or each component characteristic

8 peak intensity.

\subsection{Statistical analysis}

11 The results were presented as the mean \pm standard deviation of the mean. The slope and intercept of the curves were estimated via least-squares regression using GraphPad Prism software (ver. 8; GraphPad, Inc. San Diego, CA, USA).

\section{Results and discussion}

\subsection{Preparation and characterization of DCF-loaded HA films}

17 In this study, we first prepared a drug-polymer film as a simple model. MW and concentration of HA directly affect the solution viscosity; therefore, we chose a relatively low MW (50,000-110,000 Da) and 
1 HA films at ratios of 1:5, 1:10, 1:20, and 1:40 were approximately $16.7,9.1,4.8$, and $2.4 \%$ by weight,

2 respectively. Fig. 1 shows the digital microscopic images of the DCF-loaded HA films. At the DCF concentration range of 4.8 to $16.7 \%(\mathrm{w} / \mathrm{w})$, uniform surface crystallization was observed, and a concentration-dependent increase in surface crystallization was observed. However, at a DCF concentration of $2.4 \%$, visible DCF crystals were observed. In contrast to the crystallization observed in the films with a concentration greater than $4.8 \%$, where fine crystals grew from a significant number of nuclei, the crystals in the $2.4 \%$ film grew from a limited number of nuclei. HPLC for the quantification of DCF content in HA films $(2.4,4.8,9.1$ and $16.7 \%(\mathrm{w} / \mathrm{w}))$ showed $0.42 \pm 0.05,0.98 \pm 0.12,1.98 \pm$ 0.12 and $3.55 \pm 0.29 \mathrm{mg} /$ film, respectively. Linearity was evaluated by assessing the slope, intercept, and coefficient of determination $\left(r^{2}\right)$ for the linear fit of the quantitative value (mg/film) as a function of the theoretical drug loading ratio (\%): $y=0.218 x-0.068, r^{2}=0.999$. These results indicated that linearity was observed at certain mixing ratios of DCF in HA films.

To investigate the crystallinity of DCF after loading into the HA polymer matrices, PXRD analysis was performed (Fig. 2). XRD patterns of the reference reagent DCF powder were in agreement with those reported previously [28]. The non-drug-loaded HA film showed a harrow-amorphous pattern. In contrast, the XRD patterns of the DCF-loaded HA films showed multiple sharp peaks, indicating crystalline DCF in the films. Different peak positions of XRD patterns in the DCF-loaded HA films suggested different physical and/or chemical states of DCF in the solids. It has been reported that DCF acid, DCF, and their hydrates crystallize on several crystal forms depending on the preparation method. 
1 For example, Muangsin et al. reported that the crystal structure obtained for DCF recrystallized from

2 the matrix is DCF pentahydrate [29]. The available XRD patterns of DCF, DCF pentahydrate [28] and 3 two polymorphic forms (monoclinic $\mathrm{P} 2{ }_{1} / \mathrm{c}$ and monoclinic $\mathrm{C} 2 / \mathrm{c}$ ) of DCF acid [30], however, did not match those of the DCF-loaded HA films in our study.

\subsection{Standard Raman spectra of each component}

The first step was to determine the reference Raman spectra of DCF and HA. The Raman spectra of DCF and HA corresponded to those reported previously (Fig. 3) [31, 32]. In the Raman spectra of DCF, the characteristic peak at $1579 \mathrm{~cm}^{-1}$ was assigned to the asymmetric stretching vibration of the carboxylate. In the Raman spectra of HA, the characteristic peak at $1376 \mathrm{~cm}^{-1}$ was assigned to the $\mathrm{C}-\mathrm{H}$ bend vibration. The characteristic and non-overlapping Raman peaks enabled the identification of HA and DCF amount required for the following experiments.

\subsection{Evaluation of DCF-loaded HA films using a Raman fiber probe}

Next, we examined the relationship between the DCF content and Raman spectra. Fig. 4A shows the

Raman spectra of HA films loaded with various amounts of DCF, obtained using a Raman fiber probe.

We utilized the spherical lens Raman probe because it allowed highly sensitive and reproducible 
1 characteristic peak at $1376 \mathrm{~cm}^{-1}$ (Fig.4B) indicated a linear increase in the peak intensity with the

2 theoretical drug loading ratio (\%): $y=0.1013 x-0.0490$. These results suggested that Raman spectroscopy is feasible for the identification and quantification of DCF in HA films despite possible differences in DCF spectra depending on the physical state.

The results obtained for the films loaded with 2.4\% DCF are not included in the Fig. 4 because of the large variation in the peak intensity. Reproducible Raman spectra were obtained from 4.8 to $16.7 \%$ (w/w) DCF, but not at $2.4 \%(\mathrm{w} / \mathrm{w})$ (data not shown). It is possible that the visually observable large crystal appearing on the surface of the lower dose DCF films induces a large variation in the intensity of the DCF-specific Raman peak depending on the measurement area. This technical problem can be solved by obtaining data from a wider area [33].

\subsection{Chemical imaging analysis of DCF-loaded HA films by confocal Raman microscopy}

To examine the spatial distribution patterns of DCF in the films, two-dimensional Raman imaging analysis was performed using confocal Raman microscopy. Fig. 5A-D and E-H show optical and Raman images of DCF-loaded films, respectively, obtained by CLS. The films containing intermediate to high amounts of DCF (Fig. 5E-G) showed an increasing number of small DCF crystals on their surface with increasing DCF content. In contrast, the films containing lower amounts of DCF (Fig. 5H) showed an uneven distribution of DCF, potentially due to large or agglomerated particles, which is consistent with the optical image and Raman fiber probe results. These results demonstrate the applicability of Raman 
1 imaging analysis to characterize the spatial distribution of APIs in HA-based films.

\subsection{Fabrication and pharmaceutical characterization of DCF-loaded DMNAs}

4

We fabricated HA-based DMNAs loaded with varying amounts of DCF using micromolding technology to evaluate the applicability of Raman imaging and assess the spatial distribution of the API in DMNA needles. Fig. 6A-C shows a micrograph of the non-drug-loaded DMNA using a digital microscope. The resulting tapered cone DMNA was uniform in size (approximately $800 \mu \mathrm{m}$ in length) with sharp tips, which allows SC penetration without apparent pain [34].

We prepared four different concentrations $(2.4,4.8,9.1$, and $16.7 \%(\mathrm{w} / \mathrm{w}))$ of DCF-loaded DMNA in the same way. However, the addition of DCF induced bending of some needles during the process in a concentration-dependent manner (data not shown), which suggested a compromise in the strength of DMNA needles, as reported by Donnelly et al. [35]. For this purpose, DMNAs prepared using the same procedure were used for their characterization. HPLC analysis indicated that the amount of the drug $(2.4$, 4.8, 9.1, and $16.7 \%(\mathrm{w} / \mathrm{w}))$ in DMNA needles was $0.0074 \pm 0.0026,0.0516 \pm 0.0150,0.1789 \pm 0.0554$, and $0.3591 \pm 0.0174 \mathrm{mg} /$ array DCF, respectively.

\subsection{Chemical imaging analysis of DCF-loaded DMNA using confocal Raman microscopy}

Finally, we performed Raman imaging analysis to evaluate the spatial distribution of DCF in DMNAs.

To locate a single needle in the lateral direction ( $Y$-axis), we attached DMNA to a stationary $90^{\circ}$ edge 
1 with double-sided adhesive tape (Fig. 7). The optical and Raman images of DMNAs with and without

$2 \quad 16.7 \%$ DCF $(\mathrm{w} / \mathrm{w})$ were successfully collected over a large area enough to hold a single needle. The image showed a high Raman intensity of the HA-specific peak $\left(1376 \mathrm{~cm}^{-1}\right)$, indicating a broad distribution of HA on the surface of the needle prepared without DCF. In contrast, HA was scattered on the surface of the DCF-loaded DMNA needle. The Raman image of the high DCF characteristic peak area $\left(1579 \mathrm{~cm}^{-1}\right)$ indicated the distribution of DCF on the surface of the DCF-loaded DMNA needle. The absence of added DCF resulted in a negligible Raman peak. Overlapped figures indicate the distribution of DCF on the surface of HA-based needles.

The results indicated the applicability of the Raman imaging to assess drug distribution in DMNAs, which is relevant to ensure the safety and efficacy of formulations. The availability of the drug position in the needles is the apparent advantage of Raman imaging over other methods (e.g., use of fluorescent tracer). Combinations of Raman imaging with certain quantitative methods (e.g., HPLC) would provide valuable information for the development and production of DMNA products. This method may also be valuable for developing complex DMNA formulations that control drug distribution in various ways (e.g., use of drug-loaded microparticles and drug loading to the tip of a microneedle) [36].

Obtaining appropriate spectral data and assessing them is a major challenge for the anticipated broad application of this method. The complex shape of the small needles makes precise analysis challenging owing to difficulties in setting the appropriate focus in the measurement area, which requires an autofocusing system. Improving the data processing method to obtain the physical properties of the 
1 components (e.g., crystallinity, crystal form) is another challenge, which would increase the available

2 information for the analysis.

\section{Conclusion}

Our results suggest that Raman spectroscopy is a useful tool for determining the spatial distribution

6 of drugs in DMNAs. Availability of the distribution information in the formulation would be an apparent

7 advantage over other methods (e.g., use of fluorescent tracer, HPLC), which is valuable for the

8 development and manufacturing (e.g., troubleshooting) of DMNA products.

10 5. Declarations

11 Ethics approval and consent to participate: Not applicable

12 Consent for publication: Not applicable

13 Availability of data and material: The datasets generated during and/or analysed during the current

14 study are available from the corresponding author on reasonable request.

15 Code availability: Not applicable

16 Conflicts of interest: The authors declare that they have no conflict of interest.

17 Competing interests: The authors declare that they have no competing interests.

Funding: This research was supported by JSPS KAKENHI Grant Number JP20K16067 from the Japan

Society for the Promotion of Science (JSPS). 
1 Authors' contributions: D.A. designed the study, performed experiments, and wrote the manuscript.

2 T.M. and T.K. assisted with writing the manuscript. E.Y. and K.I. directed the research and assisted in

3 writing and editing the manuscript. All the authors have provided comments on the manuscript.

4 Acknowledgments: The authors would like to thank Fumio Kamiyama, Kenji Kajiyama and Ying-

5 Shu Quan from CosMED Pharmaceutical Co. Ltd. for useful discussions.

6 Authors' information (optional): Not applicable

8 6. References

9 1. Prausnitz MR, Langer R. Transdermal drug delivery. Nat Biotechnol. 2008;26(11):1261-1268.

10 2. Rzhevskiy AS, Singh TRR, Donnelly RF, Anissimov YG. Microneedles as the technique of drug delivery enhancement in diverse organs and tissues. J Control Release. 2018;270:184-202.

3. Depelsenaire ACI, Meliga SC, McNeilly CL, Pearson FE, Coffey JW, Haigh OL, Flaim CJ, Frazer IH, Kendall MAF. Colocalization of cell death with antigen deposition in skin enhances vaccine immunogenicity. J Invest Dermatol. 2014;134(9):2361-2370. comparative study of various protein antigen and adjuvant encapsulated nanoparticles. J Control Release. 2017;266:109-118. 
Skountzou I. Transdermal influenza immunization with vaccine-coated microneedle arrays.

$2 \quad$ PLoS One. 2009;4(3):e4773.

3 6. Matsuo K, Yokota Y, Zhai Y, Quan YS, Kamiyama F, Mukai Y, Okada N, Nakagawa S. A lowinvasive and effective transcutaneous immunization system using a novel dissolving microneedle array for soluble and particulate antigens. J Control Release. 2012;161(1):10-17.

7. Larrañeta E, Lutton REM, Woolfson AD, Donnelly RF. Microneedle arrays as transdermal and intradermal drug delivery systems: Materials science, manufacture and commercial development. Materials Science and Engineering: R: Reports. 2016;104:1-32.

8. Ito Y, Yoshimura M, Tanaka T, Takada K. Effect of lipophilicity on the bioavailability of drugs after percutaneous administration by dissolving microneedles. J Pharm Sci. 2012;101(3):11451156.

9. Ono A, Ito S, Sakagami S, Asada H, Saito M, Quan YS, Kamiyama F, Hirobe S, Okada N. Development of Novel Faster-Dissolving Microneedle Patches for Transcutaneous Vaccine Delivery. Pharmaceutics. 2017;9(3).

10. Al-Zahrani S, Zaric M, McCrudden C, Scott C, Kissenpfennig A, Donnelly RF. Microneedlemediated vaccine delivery: harnessing cutaneous immunobiology to improve efficacy. Expert Opin Drug Deliv. 2012;9(5):541-550.

11. Hiraishi Y, Nakagawa T, Quan YS, Kamiyama F, Hirobe S, Okada N, Nakagawa S. Performance and characteristics evaluation of a sodium hyaluronate-based microneedle patch for a 
2 12. Kolluru C, Gomaa Y, Prausnitz MR. Development of a thermostable microneedle patch for polio

transcutaneous drug delivery system. Int J Pharm. 2013;441(1-2):570-579.

vaccination. Drug Deliv Trans1 Res. 2019;9(1):192-203.

13. Lee KJ, Jeong SS, Roh DH, Kim DY, Choi HK, Lee EH. A practical guide to the development of microneedle systems - In clinical trials or on the market. Int J Pharm. 2020;573:118778.

14. Norman JJ. Scientific considerations for microneedle drug products: product development, manufacturing, and quality control. In.; 2016.

15. Sullivan SP, Murthy N, Prausnitz MR. Minimally invasive protein delivery with rapidly dissolving polymer microneedles. Adv Mater. 2008;20(5):933-938.

16. Katsumi H, Tanaka Y, Hitomi K, Liu S, Quan YS, Kamiyama F, Sakane T, Yamamoto A. Efficient Transdermal Delivery of Alendronate, a Nitrogen-Containing Bisphosphonate, Using Tip-Loaded Self-Dissolving Microneedle Arrays for the Treatment of Osteoporosis. Pharmaceutics. 2017;9(3).

17. Ito Y, Hirono M, Fukushima K, Sugioka N, Takada K. Two-layered dissolving microneedles formulated with intermediate-acting insulin. Int J Pharm. 2012;436(1-2):387-393.

18. Kim JY, Han MR, Kim YH, Shin SW, Nam SY, Park JH. Tip-loaded dissolving microneedles for transdermal delivery of donepezil hydrochloride for treatment of Alzheimer's disease. Eur J Pharm Biopharm. 2016;105:148-155.

19. Wang Q, Yao G, Dong P, Gong Z, Li G, Zhang K, Wu C. Investigation on fabrication process 
of dissolving microneedle arrays to improve effective needle drug distribution. Eur J Pharm Sci. 2015;66:148-156.

20. Monkare J, Pontier M, van Kampen EEM, Du G, Leone M, Romeijn S, Nejadnik MR, O'Mahony C, Slutter B, Jiskoot W, Bouwstra JA. Development of PLGA nanoparticle loaded dissolving microneedles and comparison with hollow microneedles in intradermal vaccine delivery. Eur J Pharm Biopharm. 2018;129:111-121.

21. Gordon KC, McGoverin CM. Raman mapping of pharmaceuticals. Int J Pharm. 2011;417(12):151-162.

22. Esmonde-White KA, Cuellar M, Uerpmann C, Lenain B, Lewis IR. Raman spectroscopy as a process analytical technology for pharmaceutical manufacturing and bioprocessing. Anal Bioanal Chem. 2017;409(3):637-649.

23. Allan P, Bellamy LJ, Nordon A, Littlejohn D, Andrews J, Dallin P. In situ monitoring of powder blending by non-invasive Raman spectrometry with wide area illumination. Journal of Pharmaceutical and Biomedical Analysis. 2013;76:28-35.

24. Šašić S, Clark DA, Mitchell JC, Snowden MJ. A comparison of Raman chemical images produced by univariate and multivariate data processing — a simulation with an example from pharmaceutical practice. The Analyst. 2004;129(11):1001-1007.

25. Farias MAdS, Soares FLF, Carneiro RL. Crystalline phase transition of ezetimibe in final product, after packing, promoted by the humidity of excipients: Monitoring and quantification 
by Raman spectroscopy. Journal of Pharmaceutical and Biomedical Analysis. 2016;121:209-

26. Altman R, Bosch B, Brune K, Patrignani P, Young C. Advances in NSAID development: evolution of diclofenac products using pharmaceutical technology. Drugs. 2015;75(8):859-877.

27. Hirobe S, Azukizawa H, Hanafusa T, Matsuo K, Quan YS, Kamiyama F, Katayama I, Okada N, Nakagawa S. Clinical study and stability assessment of a novel transcutaneous influenza vaccination using a dissolving microneedle patch. Biomaterials. 2015;57:50-58.

28. Muangsin N, Prajuabsook M, Chimsook P, Chantarasiri N, Siraleartmukul K, Chaichit N, Hannongbua S. Structure determination of diclofenac in a diclofenac - containing chitosan matrix using conventional X - ray powder diffraction data. Journal of Applied Crystallography. $2004 ; 37(2)$.

29. Muangsin N, Prajaubsook M, Chaichit N, Siritaedmukul K, Hannongbua S. Crystal Structure of a Unique Sodium Distorted Linkage in Diclofenac Sodium Pentahydrate. Analytical Sciences. 2002;18(8):967-968.

30. Korkiatithaweechai S, Umsarika P, Praphairaksit N, Muangsin N. Controlled release of diclofenac from matrix polymer of chitosan and oxidized konjac glucomannan. Mar Drugs. 2011;9(9):1649-1663.

31. Essendoubi M, Gobinet C, Reynaud R, Angiboust JF, Manfait M, Piot O. Human skin penetration of hyaluronic acid of different molecular weights as probed by Raman spectroscopy. 
2 32. Fini A, Cavallari C, Ospitali F. Diclofenac Salts. V. Examples of Polymorphism among Diclofenac Salts with Alkyl-hydroxy Amines Studied by DSC and HSM. Pharmaceutics. 2010;2(2):136-158

5 33. Shin K, Chung H. Wide area coverage Raman spectroscopy for reliable quantitative analysis and its applications. Analyst. 2013;138(12):3335-3346.

7 34. Gill HS, Denson DD, Burris BA, Prausnitz MR. Effect of microneedle design on pain in human volunteers. Clin J Pain. 2008;24(7):585-594.

9 35. Donnelly RF, Morrow DI, Singh TR, Migalska K, McCarron PA, O'Mahony C, Woolfson AD. Processing difficulties and instability of carbohydrate microneedle arrays. Drug Dev Ind Pharm. 2009;35(10):1242-1254. techniques of microneedles. Drug Deliv Transl Res. 2018;8(6):1828-1843. 
1

\section{Figure legends}

2 Fig 1 Optical image of DCF-loaded HA films, A) 0\%, B) 2.4\%, C) 4.8\%, D) 9.1\% and E) $16.7 \%$ DCF

$3(\mathrm{w} / \mathrm{w})$. The scale bars are $2000 \mu \mathrm{m}$

4

Fig 2 PXRD patterns of DCF-loaded HA films and reference materials, A) HA, B) DCF, C) 0\%, D)

5

$2.4 \%$, E) $4.8 \%$, F) $9.1 \%$ and G) $16.7 \%$ DCF (w/w)

6

Fig 3 Raman spectra of reference materials, A) HA and B) DCF. Asterisk and double asterisk indicate the HA characteristic peak at $1376 \mathrm{~cm}^{-1}$ and DCF characteristic peak at $1579 \mathrm{~cm}^{-1}$

8

9

10

Fig 4 (A) Raman spectra of DCF-loaded HA films obtained using fiber probe Raman, a) 16.7\%, b) 9.1\%,

c) $4.8 \%$, d) $0 \% \mathrm{DCF}(\mathrm{w} / \mathrm{w})$. Gray and hatched area indicate the HA characteristic peak at $1376 \mathrm{~cm}^{-1}$ and DCF characteristic peak at $1579 \mathrm{~cm}^{-1}$. (B) The relationship between DCF weight ratio (\%) and relative Raman intensity ratio of DCF characteristic peak at $1579 \mathrm{~cm}^{-1}$ to HA characteristic peak at $1376 \mathrm{~cm}^{-1}$.

Each point represents the mean $\pm \mathrm{SD}(\mathrm{n}=4)$

Fig 5 Raman spectroscopy mapping of DCF-loaded HA film, Optical images (A-D) and Raman images generated by CLS (E-H). A) and E) 16.7\%, B) and F) 9.1\%, C) and G) 4.8\%, D) and H) $2.4 \%$ DCF (w/w). Green and blue colors represent DCF and HA. The scale bars are $200 \mu \mathrm{m}$

Fig 6 The optical image of dissolving microneedle array

Fig 7 Raman spectroscopy mapping of DCF-loaded DMNA, non-drug-loaded DMNA (A-D), 16.7\% DCF (w/w) -loaded DMNA (E-H), optical images (A and E), Raman images generated by HA characteristic peak intensity at $1376 \mathrm{~cm}^{-1}$ (blue, B and F), DCF characteristic peak intensity at $1579 \mathrm{~cm}^{-}$ 
$1{ }^{1}$ (green, $\mathrm{C}$ and $\left.\mathrm{G}\right)$, and merge (D and $\left.\mathrm{H}\right)$. The scale bars are $50 \mu \mathrm{m}$ 
Figures
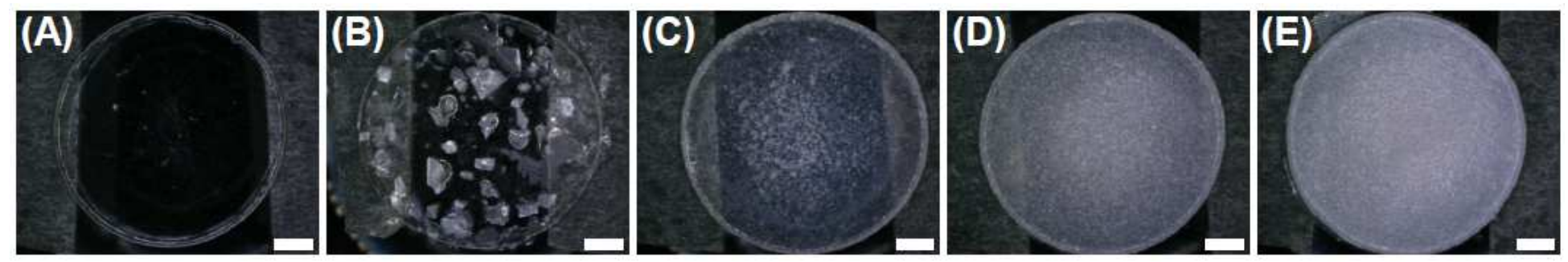

Figure 1

Optical image of DCF-loaded HA films, A) 0\%, B) $2.4 \%, C) 4.8 \%$, D) $9.1 \%$ and E) $16.7 \%$ DCF (w/w). The scale bars are $2000 \mu \mathrm{m}$

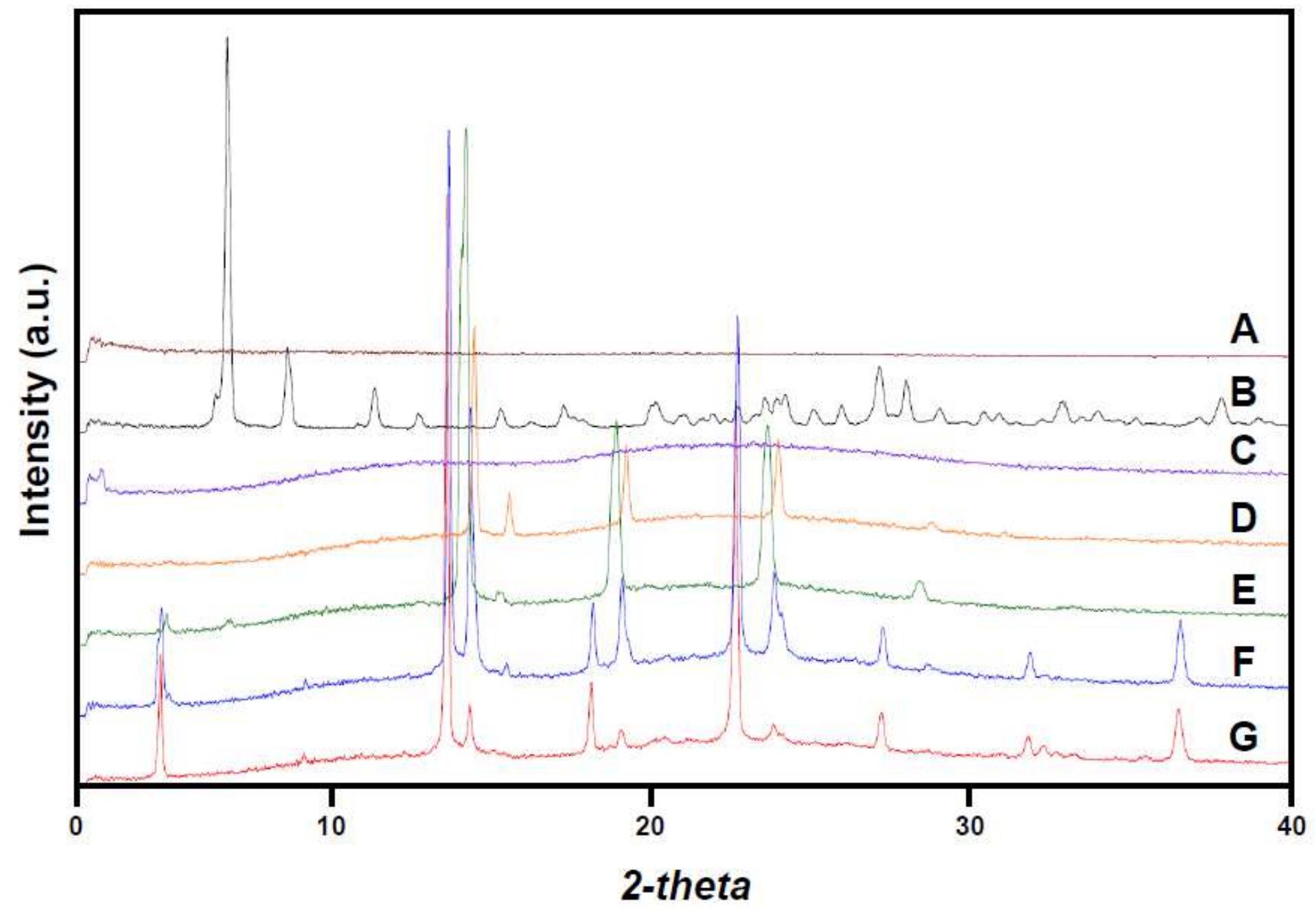

Figure 2

PXRD patterns of DCF-loaded HA films and reference materials, A) HA, B) DCF, C) $0 \%$, D) $2.4 \%, E) 4.8 \%, F$ ) 9.1\% and G) $16.7 \%$ DCF $(w / w)$ 


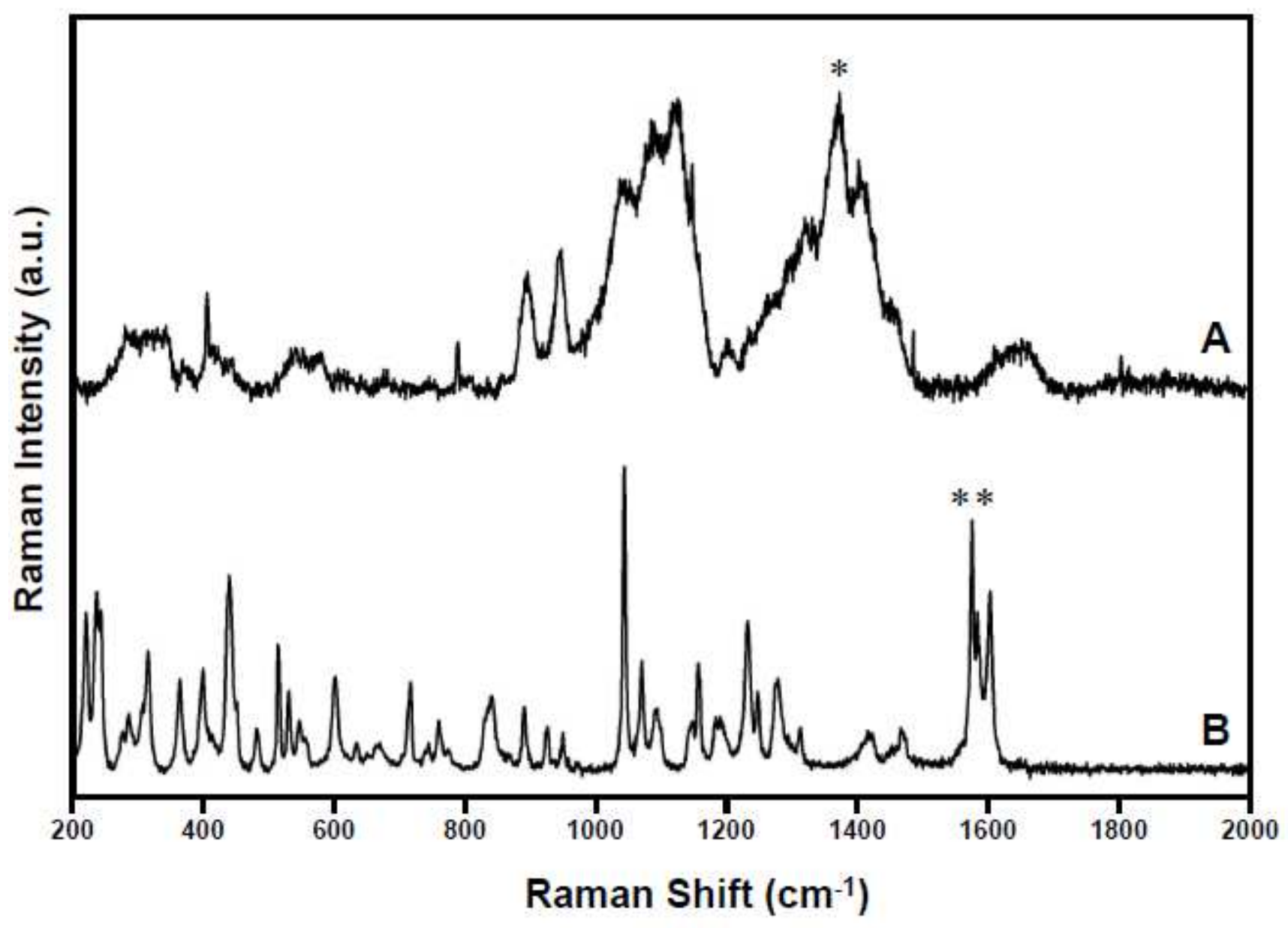

Figure 3

Raman spectra of reference materials, A) HA and B) DCF. Asterisk and double asterisk indicate the HA characteristic peak at $1376 \mathrm{~cm}-1$ and DCF characteristic peak at $1579 \mathrm{~cm}-1$ 

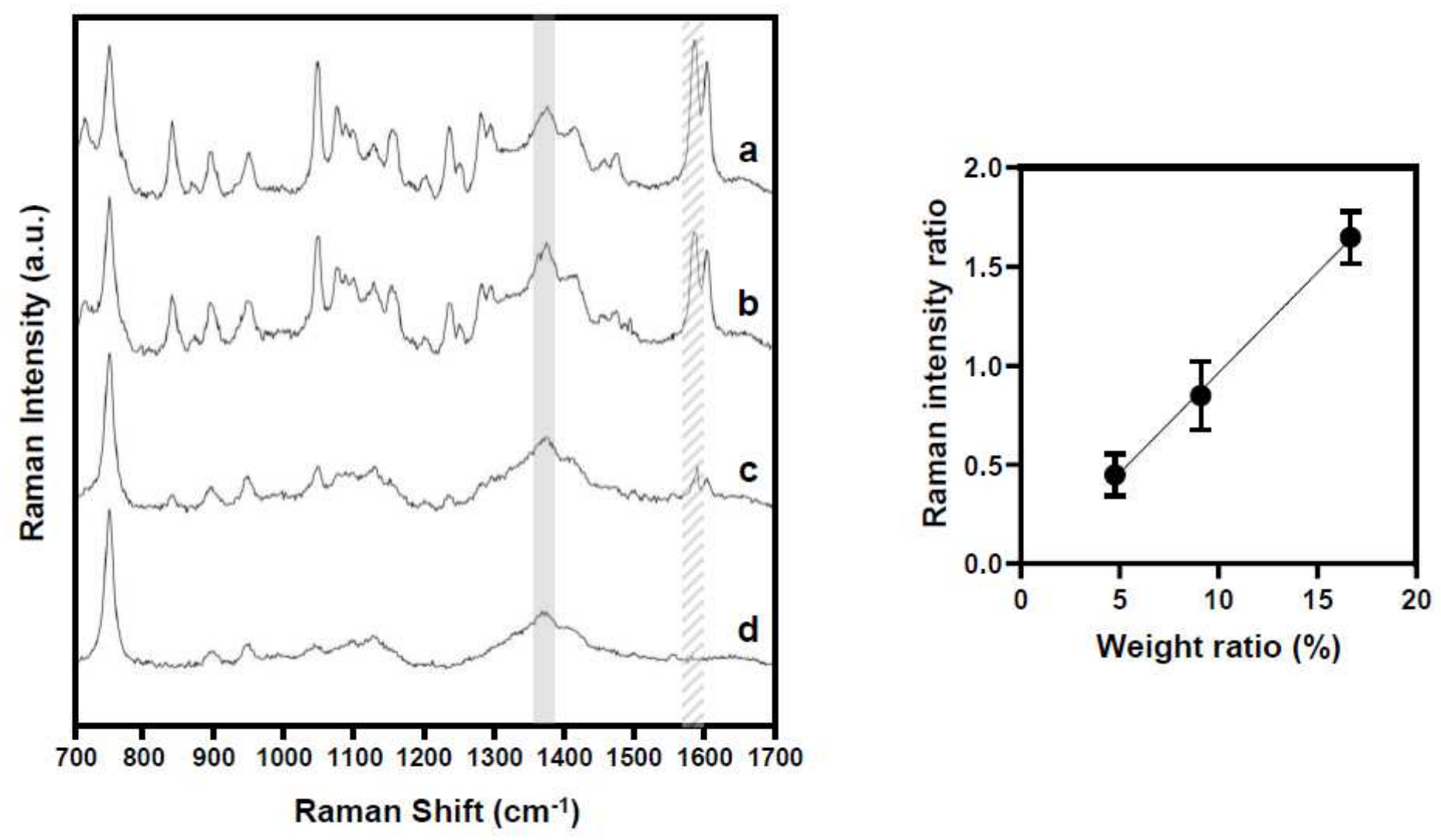

Figure 4

(A) Raman spectra of DCF-loaded HA films obtained using fiber probe Raman, a) $16.7 \%$, b) $9.1 \%$, c) $4.8 \%$, d) $0 \%$ DCF (w/w). Gray and hatched area indicate the HA characteristic peak at $1376 \mathrm{~cm}-1$ and DCF characteristic peak at $1579 \mathrm{~cm}-1$. (B) The relationship between DCF weight ratio (\%) and relative Raman intensity ratio of DCF characteristic peak at $1579 \mathrm{~cm}-1$ to HA characteristic peak at $1376 \mathrm{~cm}-1$. Each point represents the mean SD $(n=4)$ 

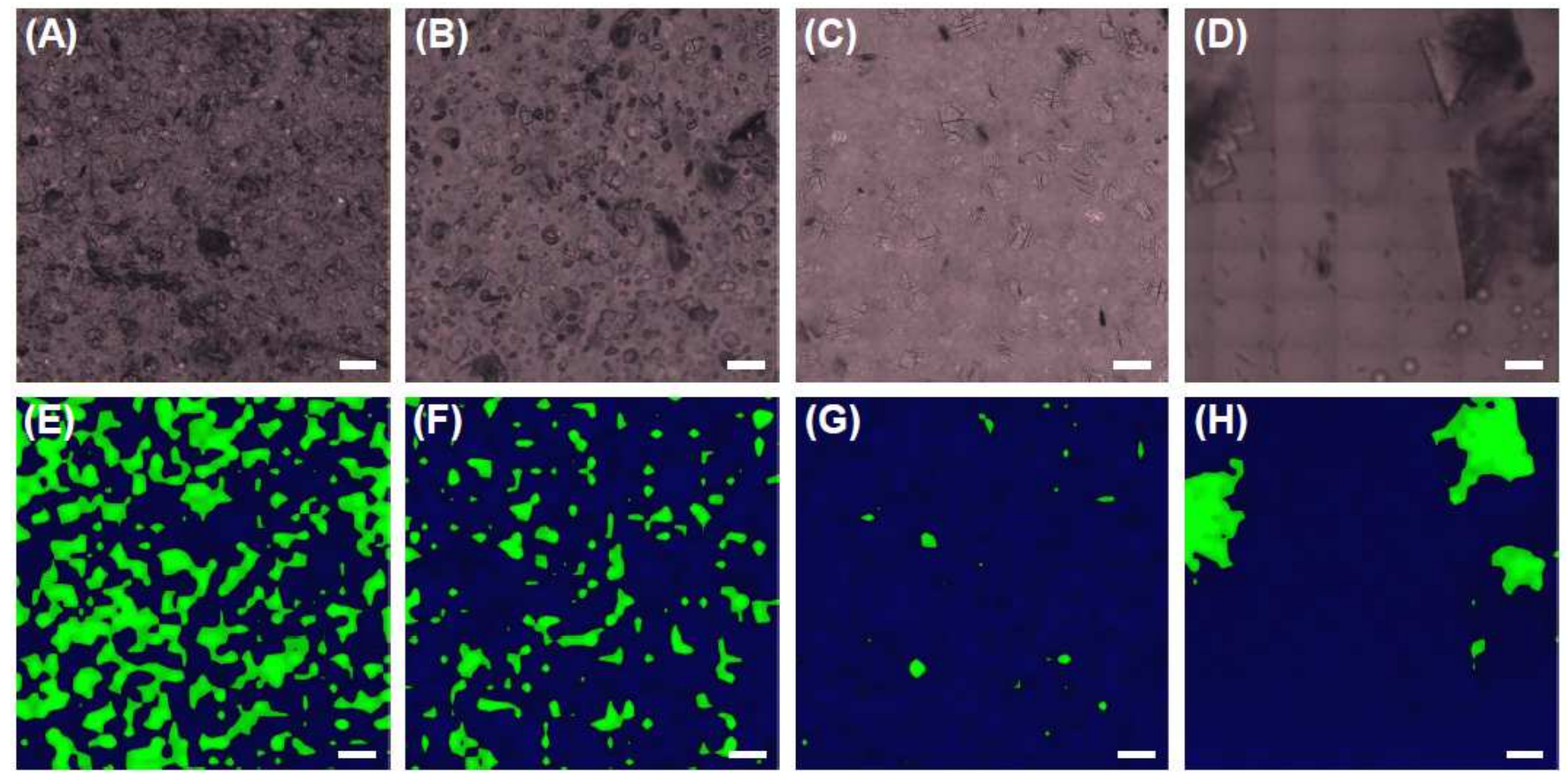

Figure 5

Raman spectroscopy mapping of DCF-loaded HA film, Optical images (A-D) and Raman images generated by CLS (E-H). A) and E) $16.7 \%, B)$ and F) $9.1 \%, C)$ and $G) 4.8 \%, D)$ and $H) 2.4 \%$ DCF (w/w). Green and blue colors represent DCF and HA. The scale bars are $200 \mu \mathrm{m}$
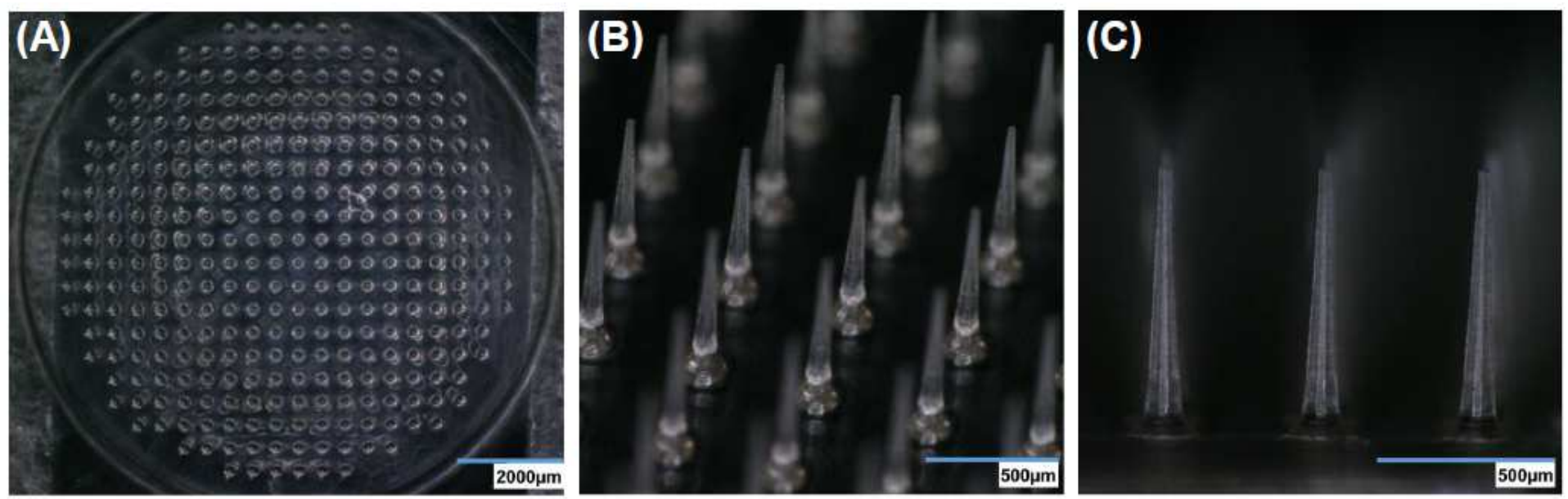

\section{Figure 6}

The optical image of dissolving microneedle array 


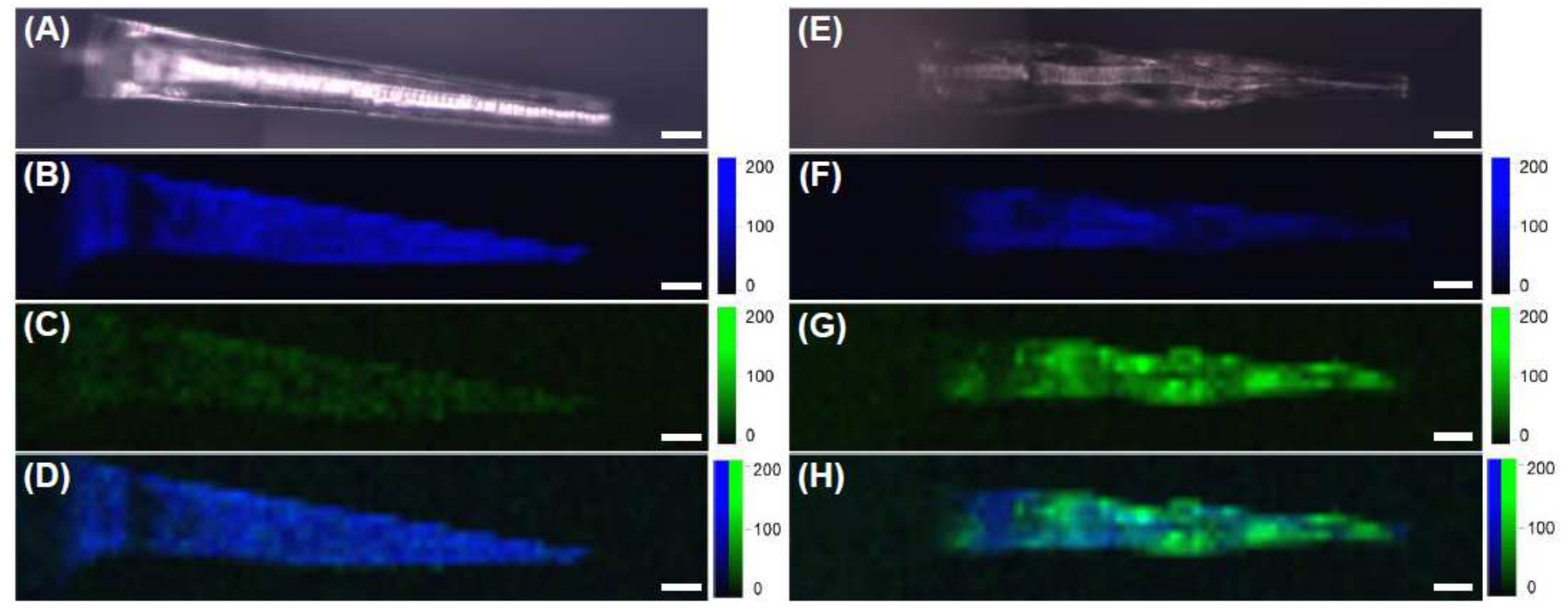

Figure 7

Raman spectroscopy mapping of DCF-loaded DMNA, non-drug-loaded DMNA (A-D), 16.7\% DCF (w/w) loaded DMNA (E-H), optical images (A and E), Raman images generated by HA characteristic peak intensity at $1376 \mathrm{~cm}-1$ (blue, B and F), DCF characteristic peak intensity at $1579 \mathrm{~cm}-1$ (green, C and G), and merge $(\mathrm{D}$ and $\mathrm{H})$. The scale bars are $50 \mu \mathrm{m}$

\section{Supplementary Files}

This is a list of supplementary files associated with this preprint. Click to download.

- GraphicalAbstract.png 